\title{
A Hybrid Polyhedral Uncertainty Model for the Robust Network Loading Problem
}

\author{
Ayşegül Altın, Hande Yaman, and Mustafa Ç. Pınar
}

\section{Introduction}

For a given undirected graph $G$, the network loading problem (NLP) deals with the design of a least cost network by allocating discrete units of capacitated facilities on the links of $G$ so as to support expected pairwise demands between some endpoints of $G$. For a telephone company, the problem would be to lease digital facilities for the exclusive use of a customer where there is a set of alternative technologies with different transmission capacities. For example, DS0 is the basis for digital multiplex transmission with a signalling rate of 64 bits per second. Then, DS1 and DS3 correspond to 24 and 672 DS0s in terms of transmission capacities, respectively. The cost of this private service is the total leasing cost of these facilities, which is determined in a way to offer significant economies of scale. In other words, the least costly combination of these facilities would be devoted to a single customer to ensure communication between its sites. Then the customer would pay just a fixed amount for leasing these facilities and would not make any additional payment in proportion to the amount of traffic its sites exchange with each other. The structure of the leasing cost, which offers economies of scale, complicates the problem [31].

Although the traditional approach is to assume that the customer would be able to provide accurate estimates for point-to-point demands, this is not very likely to happen in real life. Hence, we relax this assumption and study the robust NLP to obtain designs flexible enough to accommodate foreseen fluctuations in demand.

\footnotetext{
Ayşegül Altın

Department of Industrial Engineering, TOBB University of Economics and Technology, Söğütözü 06560 Ankara, Turkey

e-mail: aaltin@etu.edu.tr

Hande Yaman

Department of Industrial Engineering, Bilkent University, 06800 Ankara, Turkey

e-mail: hyaman@bilkent.edu.tr

Mustafa Ç. Pınar

Department of Industrial Engineering, Bilkent University, 06800 Ankara, Turkey

e-mail: mustafap@bilkent.edu.tr
} 
Our aim is to design least cost networks which remain operational for any feasible realization in a prescribed demand polyhedron.

Efforts for incorporating uncertainty in data can be divided into two main categories. The first one is stochastic optimization(SO), where data are represented as random variables with a known distribution and decisions are taken based on expectations. However, it is computationally quite challenging to quantify these expectations. Moreover, given that limited or no historical data are available most of the time, it is not realistic to assume that exact distributions can be obtained reliably. Besides, SO yields decisions that might become infeasible with some probability. This latter issue might lead to undesirable situations especially when such a tolerance is not preferable. Alternatively, in robust optimization (RO) data are represented as uncertainty sets like polyhedral sets and the best decision is the one with the best worst-case performance, i.e., the one to handle the worst-case scenario in the uncertainty set in the most efficient way. Besides, RO is computationally tractable for some polyhedral or conic quadratic uncertainty sets and for many classes of optimization [36]. The interested reader can refer to [4, 6, 9-14, 33, 34, 40] for several examples of RO models and methodology.

In network design, the most common component subject to uncertainty is the traffic matrix, i.e., the traffic demand between some node pairs in the network. In this chapter, we study the robust network loading problem under polyhedral uncertainty. A few polyhedral demand models have found acceptance in the telecommunications network design society. The initial efforts belong to Duffield et al. [18] and Fingerhut et al. [20], who propose the so-called hose model independently, for the design of virtual private networks (VPNs) and broadband networks, respectively. The hose model has become quickly popular since it handles complicated communication requests efficiently and scales well as network sizes continue to grow. This is mainly because it does not require any estimate for pairwise demands but defines the set of feasible demand realizations via bandwidth capacities of some endpoints called terminals in the customer network.

Later, Bertsimas and Sim [13,14] introduce an alternative demand definition where they consider lower and upper bounds on the uncertain coefficients and allow at most a fixed number of coefficients to assume their worst possible values. They suggest to use this quota as a measure for the trade-off between the conservatism and the cost of the final design. Their model, which we will refer to as the $B S$ model in the rest of the chapter, has also gained significant adherence in several applications of network design. In our network design context, the Bertsimas-Sim uncertainty definition amounts to specifying lower and upper bounds on the pointto-point communication demands and to allowing at most a fixed number of pairs to exchange their maximum permissible amount of traffic.

Finally, Ben-Ameur and Kerivin [8] propose to use a rather general demand polyhedron, which could be constructed by describing the available information for the specific network using a finite number of linear inequalities. The common point for all these demand models is that, no matter which definition you use, the concern is to determine the least costly link capacity configuration that would remain operational under the worst case. We will refer to the feasible demand realization which leads 
to the most costly capacity configuration for the optimal routing as the worst-case scenario throughout this chapter.

Against this background, our main contribution is to introduce a new demand model called the hybrid model, which specifies lower and upper bounds on the point-to-point communication demands as well as bandwidth capacities as in the hose model. In other words, the hybrid model aims to make the hose model more accurate by incorporating additional information on pairwise demands. The advantage of extra information in the form of lower and upper bounds is to avoid redundant conservatism.

NLP is an important problem, which can be applied to different contexts like private network design or capacity expansion in telecommunications, supply chain capacity planning in logistics, or truck loading in transportation. Existing studies on the deterministic problem can be grouped under several classes. One source of variety is the number of facility alternatives. Single-facility [5, 16, 30, 32, 35] and two-facility $[17,21,31]$ problems are the most common types. On the other hand, NLP with flow costs $[17,21,35]$ and without flow costs [7, 16, 29-31] are also widely studied. Although static routing is always used, the multi-path routing $[5,7,16,17,21,29-31,35]$ and single-path routing $[5,15,22]$ lead to a technical classification of the corresponding literature.

Although the deterministic NLP is widely studied, the Rob-NLP literature is rather limited. Karaşan et al. [27] study DWDM network design under demand uncertainty with an emphasis on modelling, whereas Altın et al. [3] provide a compact MIP formulation for Rob-NLP and a detailed polyhedral analysis of the problem for the hose model as well as an efficient branch-and-cut algorithm. Atamtürk and Zhang [6] study the two-stage robust NLP where the capacity is reserved on network links before observing the demands and the routing decision is made afterwards in the second stage. Furthermore, Mudchanatongsuk et al. [33] study an approximation to the robust capacity expansion problem with recourse, where the routing of demands (recourse variables) is limited to a linear function of demand uncertainty. They consider transportation cost and demand uncertainties with binary capacity design variables and show that their approximate solutions reduce the worst-case cost but incur sub-optimality in several instances.

In addition to introducing the hybrid model, we initially give a compact mixed integer programming model of Rob-NLP with an arbitrary demand polyhedron [3]. Then we focus on the hybrid model and discuss two alternative MIP models for the corresponding Rob-NLP. Next, we compare them in terms of the computational performance using an off-the-shelf MIP solver and mention the differences in terms of the polyhedral structures of their feasible sets. Moreover, we provide an experimental economic analysis of the impact of robustness on the design cost. Finally, we compare the final design cost for the hybrid model with those for the hose and BS models.

In Section 2 we first define our problem briefly. Then in Section 2.1, we introduce the hybrid model as a new, general-purpose demand uncertainty definition and present alternative MIP models. We present results of computational tests in 
Section 3. Then, we conclude the chapter with Section 4, where we summarize our results and mention some future research directions.

\section{Problem Definition}

For a given undirected graph $G=(V, E)$ with the set of nodes $V$ and the set of edges $E$, we want to design a private network among the set of customer sites $W \subseteq$ $V$. Let $Q$ be the set of commodities where each commodity $q \in Q$ corresponds to a potential communication demand from the origin site $o(q) \in W$ to the destination site $t(q) \in W \backslash\{o(q)\}$. In this chapter, our main concern is to allocate discrete number of facilities with different capacities on the edges of $G$ to design a least-cost network viable for any demand realization in a prescribed polyhedral set.

Let $L$ be the set of facility types, $C^{l}$ be the capacity of each type $l$ facility, $p_{e}^{l}$ be the cost of using one unit of type $l$ facility on link $e$, and $y_{e}^{l}$ be the number of type $l \in L$ facilities reserved on link $e$. Moreover, $d_{q}$ is the estimated demand from node $o(q)$ to node $t(q)$ whereas $f_{h k}^{q}$ is the fraction of $d_{q}$ routed on the edge $\{h, k\} \in E$ in the direction from $h$ to $k$. Throughout the chapter, we will sometimes use $\{h, k\}$ in place of edge $e$ if we need to mention the end points of $e$. Using this notation, the MIP formulation for the traditional NLP is as follows:

$$
\begin{array}{cc}
\min \sum_{e \in E} \sum_{l \in L} p_{e}^{l} y_{e}^{l} & \\
\text { s.t. } \sum_{k:\{h, k\} \in E}\left(f_{h k}^{q}-f_{k h}^{q}\right)=\left\{\begin{array}{r}
1 h=o(q) \\
-1 h=t(q) \\
0 \text { otherwise }
\end{array}\right. & \forall h \in V, q \in Q, \\
\sum_{q \in Q}\left(f_{h k}^{q}+f_{k h}^{q}\right) d_{q} \leq \sum_{l \in L} C^{l} y_{e}^{l} & \forall e=\{h, k\} \in E, \\
y_{e}^{l} \geq 0 \text { integer } & \forall l \in L, e \in E, \\
f_{h k}^{q}, f_{k h}^{q} \geq 0 & \forall\{h, k\} \in E, q \in Q .
\end{array}
$$

The main motivation of the current work is to incorporate some robustness and flexibility into the capacity configuration decision. Accordingly, we consider the possibility of changes in demand expectations and determine the least-cost design based on a polyhedral set of admissible demands rather than a single matrix of average estimates. Let $D=\left\{d \in \mathbb{R}^{|Q|}: \sum_{q \in Q} a_{z}^{q} d_{q} \leq \alpha_{z} \forall z=1, \ldots, H, d_{q} \geq 0 \forall q \in Q\right\}$ be the polyhedron containing non-simultaneous demand matrices, which are admissible given the available information about the network. The most significant impact of such an extension is observed in constraint (3), which has to be replaced with

$$
\sum_{q \in Q}\left(f_{h k}^{q}+f_{k h}^{q}\right) d_{q} \leq \sum_{l \in L} C^{l} y_{e}^{l} \forall d \in D, e=\{h, k\} \in E
$$


since we want the final capacity configuration to support any feasible realization $d \in D$. However, this leads to a semi-infinite optimization problem since we need one constraint for each of the infinitely many feasible demand matrices in $D$. To overcome this difficulty, we use a common method in robust optimization $[1,11,13]$ and obtain a compact MIP formulation of the problem. This method which was also used in [3] is now briefly summarized for the sake of completeness.

First, observe that any one of the infinitely many non-simultaneous feasible communication request $d \in D$ would be routed safely along each link if the capacity of each link is sufficient to route the most capacity consuming, i.e., the worst-case, admissible traffic requests. As a result, we can model our problem using the following semi-infinite MIP formulation $\left(N L P_{p o l}\right)$ :

$$
\begin{gathered}
\min \sum_{e \in E} \sum_{l \in L} p_{e}^{l} y_{e}^{l} \\
\text { s.t } \quad(2),(4),(5) \\
\max _{d \in D} \sum_{q \in Q}\left(f_{h k}^{q}+f_{k h}^{q}\right) d_{q} \leq \sum_{l \in L} C^{l} y_{e}^{l} \forall e=\{h, k\} \in E,
\end{gathered}
$$

where we replace (3) with (7) to ensure (6). Notice that given a routing $f$, we can obtain the worst-case capacity requirement for each link $e \in E$ by solving the linear programming problem on the left-hand side of (7). Hence for each link $e=\{h, k\} \in$ $E$, we can apply a duality-based transformation to the maximization problem in (7) and reduce $N L P_{p o l}$ to the following compact MIP formulation $\left(N L P_{D}\right)$ :

$$
\min \sum_{e \in E} \sum_{l \in L} p_{e}^{l} y_{e}^{l}
$$

s.t.

$$
\text { (2), (4), (5) }
$$

$$
\begin{aligned}
\sum_{z=1}^{H} \alpha_{z} \lambda_{e}^{z} & \leq \sum_{l \in L} C^{l} y_{e}^{l} \forall e \in E, \\
f_{h k}^{q}+f_{k h}^{q} & \leq \sum_{z=1}^{H} a_{z}^{q} \lambda_{e}^{z} \forall e=\{h, k\} \in E, q \in Q, \\
\lambda_{e}^{z} \geq 0 \quad \forall z & =1, \ldots, H, q \in Q,
\end{aligned}
$$

where $\lambda \in \mathfrak{R}^{H|E|}$ are the dual variables used in the transformation. The interested reader can refer to Altin et al. [2] for a more detailed discussion of this approach.

The above duality transformation motivates two important contributions. First, we obtain a compact MIP formulation, which we can solve for small-to-mediumsized instances using off-the-shelf MIP solvers. Moreover, we get rid of the bundle constraints (3), which complicate the polyhedral studies on traditional NLP. In Altın et al. [3], we benefit from this single-commodity decomposition property and provide a thorough polyhedral analysis for the so-called symmetric hose model of demand uncertainty. In the next section, we will introduce the hybrid model as 
a new general-purpose polyhedral demand definition and study Rob-NLP for this uncertainty set.

\subsection{The Hybrid Model}

Due to the dynamic nature of the current business environment, the variety of communication needs keeps increasing and hence it gets harder to accurately estimate point-to-point demands. Therefore, designing networks that are flexible enough to handle multiple demand scenarios efficiently so as to improve network availability has become a crucial issue. Although considering a finite number of potential scenarios is a well-known approach in stochastic optimization, Duffield et al. [18] and Fingerhut et al. [20] introduced the hose model as a first effort to use polyhedral demand uncertainty sets in telecommunications context. In its most general form, that is called the asymmetric hose model, outflow $\left(b_{s}^{+}\right)$and inflow $\left(b_{s}^{-}\right)$capacities are set for each customer site $s \in W$ as

$$
\begin{gathered}
\sum_{q \in Q: o(q)=s} d_{q} \leq b_{s}^{+} \forall s \in W, \\
\sum_{q \in Q: t(q)=s} d_{q} \leq b_{s}^{-} \forall s \in W .
\end{gathered}
$$

Then the corresponding polyhedron is $D_{\text {Asym }}=\left\{d_{q} \in \mathbb{R}^{|Q|}:\right.$ (12), (13), $d_{q} \geq$ $0 \forall q \in Q$ \}. There are also the symmetric hose model with a single capacity $b_{s}$ for the total flow that can be incident to node $s \in W$, and a sum-symmetric hose model with $\sum_{s \in W} b_{s}^{+}=\sum_{s \in W} b_{s}^{-}$.

The hose model has several strengths. To name a few, the transmission capacities can be estimated more reliably and easily than individual point-to-point demands especially if sufficient amount of statistical information is not available. Moreover, it offers resource-sharing flexibility and hence improved link utilization due to multiplexing. Basically, the size of an access link can be smaller if we use hose model rather than point-to-point lines with fixed resource sharing. These and several other competitive advantages helped the hose model to prevail within the telecommunications society [1, 3, 19, 23-26, 28, 39].

On the other hand, Bertsimas and Sim [13, 14] proposed the BS model or the restricted interval model, which defines an applicable interval for each pairwise demand such that at most a fixed number of demands can take their highest values, simultaneously. For our problem, this implies that $d_{q} \in\left[\bar{d}_{q}, \bar{d}_{q}+\hat{d}_{q}\right]$ for all $q \in Q$ and at most $\Gamma$ of these demands differ from $\bar{d}_{q} \geq 0$ at the same time. If we define each demand $q \in Q$ as $d_{q}=\bar{d}_{q}+\hat{d}_{q} \beta_{q}$ with $\beta_{q} \in\{0,1\}$, then the BS model requires $\sum_{q \in Q} \beta_{q} \leq \Gamma$. Bertsimas and Sim [14] use $\Gamma$ to control the conservatism of the final design. 
In this section, we introduce the hybrid model. Although we study a private network design problem, the hybrid model can certainly be used in any context where parameter uncertainty is a point at issue.

We call this new model as hybrid since a demand matrix $d \in \mathbb{R}^{|Q|}$ has to satisfy the slightly modified symmetric hose constraint

$$
\sum_{q \in Q: o(q)=s \bigvee t(q)=s} d_{q} \leq b_{s} \quad \forall s \in W,
$$

as well as the interval restrictions

$$
\begin{gathered}
d_{q} \leq u_{q} \forall q \in Q \\
\bar{d}_{q} \leq d_{q} \forall q \in Q
\end{gathered}
$$

to be admissible. As a result, the corresponding demand polyhedron for the hybrid model is $D_{\text {hyb }}=\left\{d \in \mathbb{R}^{|Q|}:\right.$ (14), (15), (16) $\}$. We should remark here that (14) should not be considered as analogous to the conservatism level restriction in the $B S$ model. Actually, the conservatism dimension is not articulated explicitly in the hybrid model, where the main purpose is to incorporate more information into the hose definition so as to avoid overly conservative designs taking care of unlikely worst-case demand realizations. Hence, notice that the hybrid model is a hybrid of the hose model and the interval uncertainty model but not the BS model. Finally, we are interested in the case where $D_{h y b} \neq \emptyset$ and hence $\sum_{q \in Q: o(q)=s \bigvee t(q)=s} \bar{d}_{q} \leq b_{s}$ for all $s \in W$ to have a meaningful design problem.

Let $D_{\text {sym }}=\left\{d \in \mathbb{R}^{|Q|}:\right.$ (14), $\left.d_{q} \geq 0 \forall q \in Q\right\}$. Notice that, $D_{\text {hyb }}=D_{\text {sym }}$ if $\bar{d}_{q}=0$ and $u_{q} \geq \min \left\{b_{o(q)}, b_{t(q)}\right\}$ for all $q \in Q$ whereas $D_{h y b} \subseteq D_{\text {sym }}$ otherwise. This is because we can avoid over-conservative designs and hence redundant investment by using additional information and our provisions about the specific topology.

\subsection{Robust NLP with the Hybrid Model}

In this section, we will briefly discuss two alternative MIP models for Rob-NLP with the hybrid model of demand uncertainty. The first formulation follows directly from the discussions in Section 2. On the other hand, we slightly modify our $D_{h y b}$ so as to express it in terms of deviations from the nominal values to obtain the second formulation.

First, notice that $N L P_{D}$ reduces to the following compact MIP formulation $\left(N L P_{h y b}\right)$ for the hybrid model: 


$$
\begin{array}{cl}
\min \sum_{e \in E} \sum_{l \in L} p_{e}^{l} y_{e}^{l} & \\
\text { s.t. }(2),(4),(5) & \\
\sum_{s \in W} b_{s} w_{e}^{s}+\sum_{q \in Q}\left(u_{q} \lambda_{e}^{q}-\bar{d}_{q} \mu_{e}^{q}\right) \leq \sum_{l \in L} C^{l} y_{e}^{l} \forall e \in E, & \\
w_{e}^{o(q)}+w_{e}^{t(q)}+\lambda_{e}^{q}-\mu_{e}^{q} \geq f_{h k}^{q}+f_{k h}^{q} & \forall q \in Q, e=\{h, k\} \in E, \\
\lambda_{e}^{q}, \mu_{e}^{q} \geq 0 & \forall q \in Q, e \in E, \\
w_{e}^{s} \geq 0 & \forall s \in W, e \in E,
\end{array}
$$

where $w, \lambda$, and $\mu$ are the dual variables used in the duality transformation corresponding to constraints (14), (15), and (16), respectively.

\subsection{Alternative Flow Formulation}

Given the hybrid model, we know that the best-case scenario would be the one where all demands are at their lower bounds. Then the total design cost would increase as the deviation from this best case increases. Consequently, we can restate the hybrid model in terms of the deviations from lower bounds, which requires us to modify $N L P_{p o l}$ by replacing link capacity constraints (7) with

$$
\sum_{q \in Q}\left(f_{h k}^{q}+f_{k h}^{q}\right) \bar{d}_{q}+\max _{\hat{d} \in \hat{D}_{h y b}} \sum_{q \in Q}\left(f_{h k}^{q}+f_{k h}^{q}\right) \hat{d}_{q} \leq \sum_{l \in L} C^{l} y_{e}^{l} \forall e=\{h, k\} \in E,
$$

where $\hat{D}_{h y b}=\left\{\hat{d} \in \mathbb{R}^{|Q|}: 0 \leq \hat{d}_{q} \leq \Delta_{q} \forall q \in Q ; \quad \sum_{q \in Q: o(q)=s \bigvee t(q)=s} \hat{d}_{q} \leq\right.$ $\left.\dot{b}_{s} \forall s \in W\right\}$ such that $\Delta_{q}=u_{q}-\bar{d}_{q}$ for all $q \in Q$ and $\dot{b}_{s}=b_{s}-$ $\sum_{q \in Q: o(q)=s \bigvee t(q)=s} \bar{d}_{q}$ for all $s \in W$. This observation leads to the following result.

Proposition $1 N L P D$ reduces to the following compact linear MIP formulation $\left(N L P_{\text {alt }}\right)$ for the hybrid model:

$$
\begin{aligned}
& \min \sum_{e \in E} \sum_{l \in L} p_{e}^{l} y_{e}^{l} \\
& \text { (2), (4), (5) } \\
& \sum_{q \in Q}\left(f_{h k}^{q}+f_{k h}^{q}\right) \bar{d}_{q}+\sum_{s \in W} \dot{b}_{s} v_{e}^{s}+\sum_{q \in Q} \Delta_{q} \eta_{e}^{q} \leq \sum_{l \in L} C^{l} y_{e}^{l} \forall e=\{h, k\} \in E, \\
& v_{e}^{o(q)}+v_{e}^{t(q)}+\eta_{e}^{q} \geq f_{h k}^{q}+f_{k h}^{q} \quad \forall q \in Q, e=\{h, k\} \in E, \\
& v_{e}^{o(q)}, v_{e}^{t(q)}, \eta_{e}^{q} \geq 0 \quad \forall q \in Q, e \in E .
\end{aligned}
$$


Proof For link $e=\{h, k\} \in E$, the capacity assignment $y=\left(y_{e}^{1}, \ldots, y_{e}^{|L|}\right)$ should be sufficient to route the worst-case demand as in (21). Then, we can model the maximization problem on the left-hand side of (21) as

$$
\begin{array}{cl}
\max \sum_{q \in Q}\left(f_{h k}^{q}+f_{k h}^{q}\right) \hat{d}_{q} & \\
\text { s.t. } \sum_{q \in Q: o(q)=s \bigvee t(q)=s} \hat{d}_{q} \leq \dot{b}_{s} & \forall s \in W, \\
\hat{d}_{q} \leq \Delta_{q} & \forall q \in Q, \\
\hat{d}_{q} \geq 0 & \forall q \in Q .
\end{array}
$$

Notice that for a given routing $f$, this is a linear programming problem. Since it is feasible and bounded, we can apply a duality transformation similar to Soyster [38]. So, we associate the dual variables $v_{e}^{s}$ and $\eta_{e}^{q}$ with (23) and (24), respectively, and obtain the equivalent dual formulation

$$
\begin{array}{cc}
\min \left(\sum_{s \in W} \dot{b}_{s} v_{e}^{s}+\sum_{q \in Q} \Delta_{q} \eta_{e}^{q}\right) & \\
\text { s.t. } v_{e}^{o(q)}+v_{e}^{t(q)}+\eta_{e}^{q} \geq f_{h k}^{q}+f_{k h}^{q} \forall q \in Q, & \forall s \in W, q \in Q . \\
v_{e}^{s}, \eta_{e}^{q} \geq 0 \quad & \forall s \in \mathbb{R}
\end{array}
$$

Next, we can complete the proof by equally replacing the maximization problem in (21) with (26), (27), (28) and removing min since the facility capacities $C^{l}$ and the reservation costs $p_{e}^{l}$ are nonnegative for all $l \in L$.

We show in Section 3, off-the-shelf MIP solvers can handle $N L P_{\text {alt }}$ better than $N L P_{h y b}$ in some instances.

\section{Experimental Results}

In this section, we focus on the single-facility multi-commodity problem where just one type of facility with $C$ units of capacity is available. We perform our analysis in two stages. First, we compare the performance of ILOG Cplex for the two compact MIP formulations $N L P_{h y b}$ and NLP alt in terms of solution times and bounds they provide at the end of 2-h time limit. The instances polska, dfn, newyork, france, janos, atlanta, tai, nobel-eu, pioro, and sun are from the SNDLIB web site [37] whereas the remaining are used in Altın et al. [1] for a virtual private network design problem. For the SNDLIB instances [37], we have the average demand estimates $\tilde{\tilde{d}}_{q}$. In order to generate the bandwidth values as well as the lower and upper bounds on pairwise communication demands, we have used the following relations: 
- $b_{s}=\sum_{q \in Q: o(q)=s \bigvee t(q)=s} \tilde{d}_{q}$;

- $\bar{d}_{q}=\frac{\tilde{d}_{q}}{1+p}$

- $u_{q}=(1+p) \tilde{d}_{q}$.

In our tests, we choose $p=0.2$. This parameter can be determined based on the available information about the demand pattern, past experience, etc. We should note that $D_{h y b}$ would not get smaller as $p$ increases and hence the optimal design would never get less conservative. By defining $b_{s}, u_{q}$, and $\bar{d}_{q}$ as a function of $p$, we can interpret the trade-off between the conservatism of a design and its cost. The interested reader can refer to Altın et al. [3] for an analogous parametric analysis of the symmetric hose model.

We have used AMPL to model the formulations and Cplex 11.0 MIP solver for numerical tests and set a 2-h solution time limit for all instances. We present the results of the initial comparison for two MIP models in Table 1 where we provide the following information:

- $z_{h y b}$ : best total design cost for $N L P_{h y b}$ at termination,

- $t_{c p}$ : solution time in CPU seconds for $N L P_{h y b}$,

- $G_{h y b}$ : the gap at termination for $N L P_{h y b}$,

- \# $\#_{h y b}$ : number of $B \& C$ nodes for $N L P_{h y b}$, which is 0 if no branching takes place,

- $z_{\text {alt }}$ : best total design cost for $N L P_{\text {alt }}$ at termination,

- $t_{c p}$ : solution time in CPU seconds for $N L P_{a l t}$,

- $G_{\text {alt }}$ : the gap at termination for $N L P_{\text {alt }}$,

- $\#_{\text {alt }}$ : number of $B \& C$ nodes for $N L P_{\text {alt }}$, which is 0 if no branching takes place,

- * indicates the best upper bound at termination,

- INF means that we have no integer solution at termination,

- '-' under the $z$ columns shows that even the LP relaxation cannot be solved in 2-h time limit.

We could solve both $N L P_{h y b}$ and $N L P_{\text {alt }}$ for 7 out of 17 instances to optimality within 2-h time limit. In addition to that, Cplex could also solve $N L P_{\text {alt }}$ for polska with $C=1000$. For the same instance, Cplex could reduce the integrality gap to $2.16 \%$ with $N L P_{h y b}$.

We will analyze our results in two stages. Initially, in Fig. 1, we show the reduction in solution times when $N L P_{\text {alt }}$ rather than $N L P_{h y b}$ is solved for the first seven instances in Table 1 . We measure this improvement as $\frac{t_{h y b}-t_{\text {alt }}}{t_{\text {hyb }}} \times 100$ and thus positive values show the instances that are easier to solve using the alternative formulation. We see that except $b h v 6 c$ and $p d h, N L P_{\text {alt }}$ is easier to solve for Cplex.

On the other hand, for the remaining 10 instances, we see that Cplex achieved better upper bounds with $N L P_{\text {alt }}$ in 6 cases. Figure 2 displays the termination gaps for both models. Note that for newyork, nobel-eu, and sun, Cplex could not solve even the LP relaxation of $N L P_{h y b}$ whereas we have some upper bounds for $N L P_{\text {alt }}$. We let gap $_{\text {hyb }}=105 \%$ in Fig. 2 for these three instances just for convenience. On the other hand, we see that the upper bounds on total design cost at termination are smaller with $N L P_{h y b}$ for tai and janos whereas there is a tie for polska $(C=155)$ 
Table 1 A comparison of the projected formulation and the alternative flow formulation

\begin{tabular}{llllllll}
\hline Instance & $(|V|,|E|,|W|, C)$ & $z_{\text {hyb }}$ & $t_{\text {hyb }}\left(G_{\text {hyb }}\right)$ & $\#($ hyb $)$ & $z_{\text {alt }}$ & $t_{\text {alt }}\left(G_{\text {alt }}\right)$ & $\#($ alt $)$ \\
\hline metro & $(11,42,5,24)$ & 768 & 23.02 & 428 & 768 & 13.28 & 486 \\
nsf1b & $(14,21,10,24)$ & 86,600 & 246 & 2293 & 86,600 & 134.95 & 1470 \\
at-cep1 & $(15,22,6,24)$ & 47,840 & 1.61 & 66 & 47,840 & 1.41 & 90 \\
pacbell & $(15,21,7,24)$ & 10,410 & 49.1 & 1216 & 10,410 & 28.06 & 1199 \\
bhv6c & $(27,39,15,24)$ & 810,368 & 669.09 & 13,148 & 810,368 & 725.19 & 10,960 \\
bhvdc & $(29,36,13,24)$ & 952,664 & 657.09 & 1210 & 952,664 & 149.52 & 481 \\
pdh & $(11,34,6,480)$ & $2,467,983$ & 318.41 & 4796 & $2,467,983$ & 827.58 & 10,961 \\
polska & $(12,18,12,155)$ & $44,253^{*}$ & $(0.77 \%)$ & 25,871 & $44,253^{*}$ & $(1.14 \%)$ & 19,144 \\
polska & $(12,18,12,1000)$ & $7478^{*}$ & $(2,16 \%)$ & 12,742 & 7478 & 4591.14 & 7961 \\
dfn & $(11,47,11,155)$ & $51,572^{*}$ & $(3.85 \%)$ & 4993 & $51,572^{*}$ & $(7.32 \%)$ & 281 \\
newyork & $(16,49,16,1000)$ & - & $\mathrm{INF}$ & 0 & $1,318,400^{*}$ & $(54.10 \%)$ & 100 \\
france & $(25,45,14,2500)$ & $21,600^{*}$ & $(3.22 \%)$ & 915 & $22,600^{*}$ & $(9.40 \%)$ & 151 \\
atlanta & $(15,22,15,1000)$ & $458,020,000^{*}$ & $(0.11 \%)$ & 16,023 & $458,040,000^{*}$ & $(0.36 \%)$ & 7620 \\
tai & $(24,51,19,504 \mathrm{k})$ & $28,702,323.54^{*}$ & $(20.37 \%)$ & 140 & $27,611,428.86^{*}$ & $(17.85 \%)$ & 157 \\
janos & $(26,42,26,64)$ & $1,289,931,888$ & $(99.8 \%)$ & 0 & $1,289,911,204^{*}$ & $(99.8 \%)$ & 37 \\
nobel-eu & $(28,41,28,20)$ & - & $\mathrm{INF}$ & 0 & $14,718,917,910^{*}$ & $(99.97 \%)$ & 26 \\
sun & $(27,51,24,40)$ & - & $\mathrm{INF}$ & 0 & $62,938,898.76^{*}$ & $(99.99 \%)$ & 1 \\
\hline
\end{tabular}

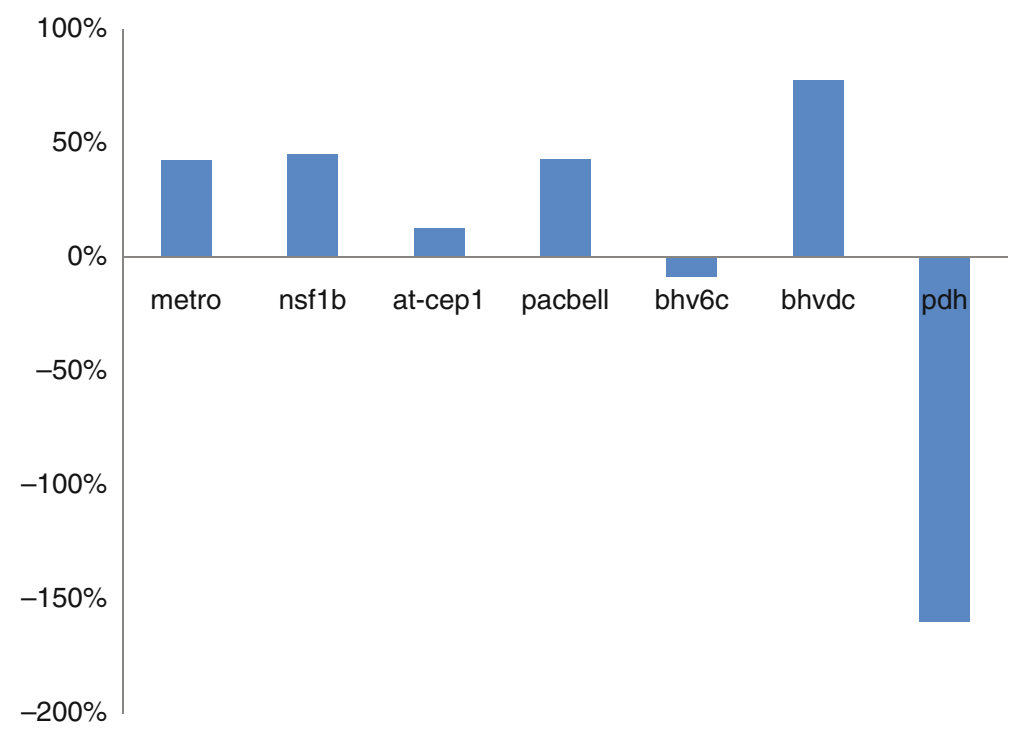

Fig. 1 Reduction in solution times when we solve $N L P_{\text {alt }}$ rather than $N L P_{h y b}$ with Cplex

and $d f n$. Based on the overall comparison of the two models that we show in Fig. 3, we can say Cplex can solve $N L P_{\text {alt }}$ more efficiently since solution times, termination gaps, and upper bounds are better with $N L P_{\text {alt }}$. On the other hand, we suppose that we can make a better use of $N L P_{h y b}$ so as to develop efficient solution tools like a branch-and-cut algorithm. We suppose $N L P_{h y b}$ to be more advantageous than $N L P_{\text {alt }}$ since the latter does not have the nice single commodity decomposition property. 


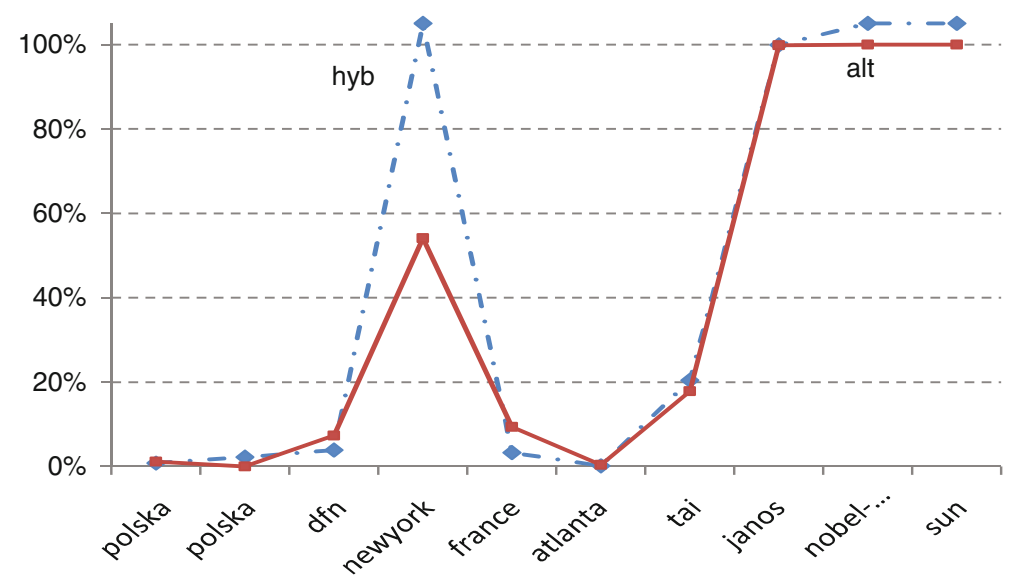

Fig. 2 Comparison of termination gaps when we solve $N L P_{\text {alt }}$ and $N L P_{h y b}$ with Cplex
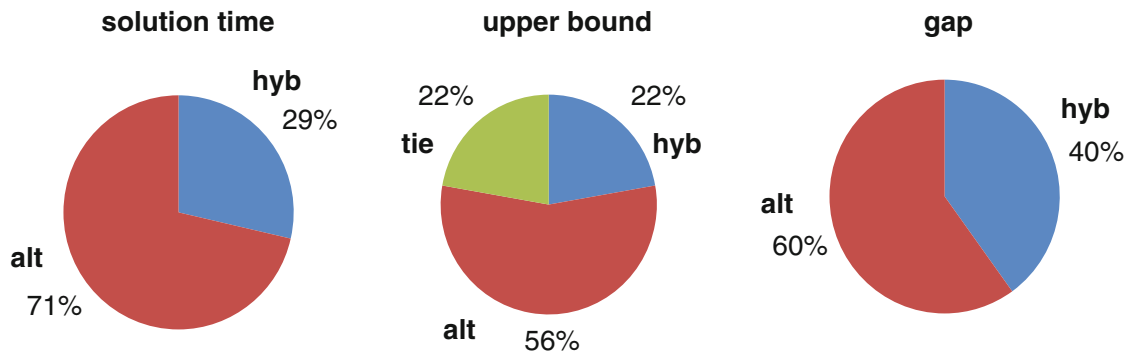

Fig. 3 A general comparison of solving $N L P_{a l t}$ and $N L P_{h y b}$ with Cplex

Next, we display how the design cost has changed according to the demand uncertainty model in Fig. 4. To this end, we consider three models: the interval uncertainty model with $D_{\text {int }}=\left\{d_{q} \in \mathbb{R}^{|Q|}: \bar{d}_{q} \leq d_{q} \leq u_{q} \forall q \in Q\right\}$, the symmetric hose model with $D_{\text {hose }}=\left\{d_{q} \in \mathbb{R}^{|Q|}:(14), d_{q} \geq 0 \forall q \in Q\right\}$, and the hybrid model. Notice that the interval model is a special case of the BS model with $\Gamma=|Q|$ and thus the corresponding worst case would be $d_{q}^{\text {worst }}=u_{q}$ for all $q \in Q$. We consider six instances, which we could solve to optimality in reasonable times for all demand models. We should remark here that we had to terminate the test for the $b h v d c$ and $b h v 6 c$ instances under interval uncertainty model after 60,000 CPU seconds with $0.21 \%$ and $0.3 \%$ gaps since the best solutions have not changed for a long while and the gaps are relatively small.

Let $z_{\text {det }}$ be the total design cost for the deterministic case if we consider the best-case scenario with $d_{q}=\bar{d}_{q}$ for all $q \in Q$. Then for the three demand models, we show the percent of increase in design cost, which is $\frac{z_{h y b}-z_{\text {det }}}{z_{\text {det }}} * 100$ for the hybrid model and similar for the other models, in Fig. 4. For each instance such 


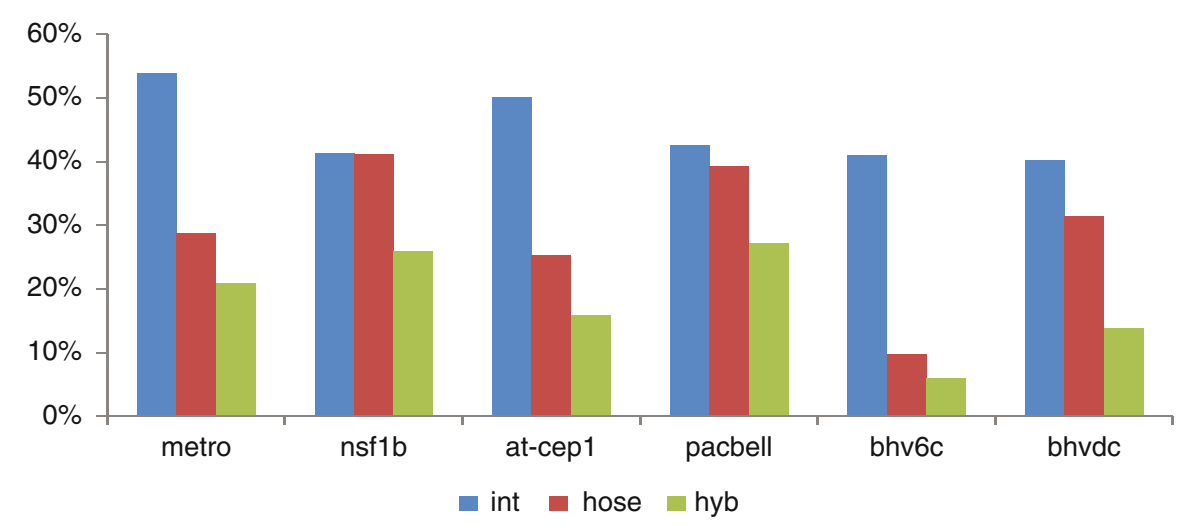

Fig. 4 Increase in design cost with respect to the deterministic case for different demand models

an increase can be interpreted as the cost of robustness or the price that we should be ready to pay so as to have a more flexible network and hence increased service availability. We see that as we shift from the interval model to the hose model and then to the hybrid model, the total design cost decreases significantly, namely, the average increase rates are $44.89 \%, 29.33 \%$, and $18.31 \%$ for these six instances with three models, respectively. Given that these instances are constructed using the same parameters $(b, \bar{d}, u) \in \mathbb{R}_{+}^{|W|+2 *|Q|}$, we can interpret this decreasing trend in cost as a consequence of using more informative demand uncertainty sets and hence being protected against practically and technically more realistic worst-case scenarios.

Our worst-case definition over a polyhedron is clearly quite different from simply determining the worst-case scenario a priori. Since we exploit the hybrid model information, we can avoid over-conservative designs. Suppose that we have not done so and we determine a worst case that can happen using the available information $(b, \bar{d}, u) \in \mathbb{R}_{+}^{|W|+2 *|Q|}$. Obviously, the safest approach would be to set $d_{q}=\min \left\{b_{o(q)}, b_{t(q)}, u_{q}\right\}$ for all $q \in Q$ and then solve the nominal problem (1), (2), (3), (4), and (5) to get the optimal design cost $z_{\text {worst }}$. When we compare the design cost $z_{\text {hyb }}$ with $z_{\text {worst }}$ for the six instances we have mentioned above, we see that the design costs have reduced by $18.27 \%$ on the average. On the other hand, the average savings is around $10.61 \%$ for the hose model. Figure 5 displays the percentage of savings in cost for each instance with both models. We also compare the design costs for the hybrid model and the $B S$ model for $\Gamma=\lceil 0.1|Q|\rceil$ and $\Gamma=\lceil 0.15|Q|\rceil$. We show the relative savings the hybrid model provide in Fig. 6 . We see that when $\Gamma=\lceil 0.1|Q|\rceil$, using the hybrid model yields a less costly design for all instances except $n s f 1 b$, where it is only $0.12 \%$ worse. On the average, the hybrid model provides $6.53 \%$ and $10.69 \%$ savings, respectively, for these six instances and the difference increases rapidly as $\Gamma$ grows larger.

Finally, we consider the metro, at-cepl, and pacbell instances so as to compare the robust designs for the $B S$ model $(\Gamma=\lceil 0.15|Q|\rceil)$ and the hybrid model in 


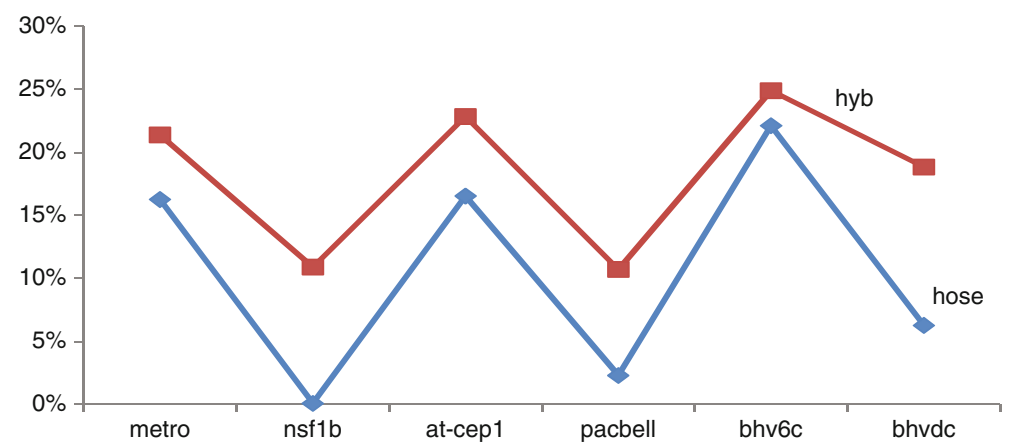

Fig. 5 Reduction in design cost with respect to the worst-case scenario determined without exploiting the demand model
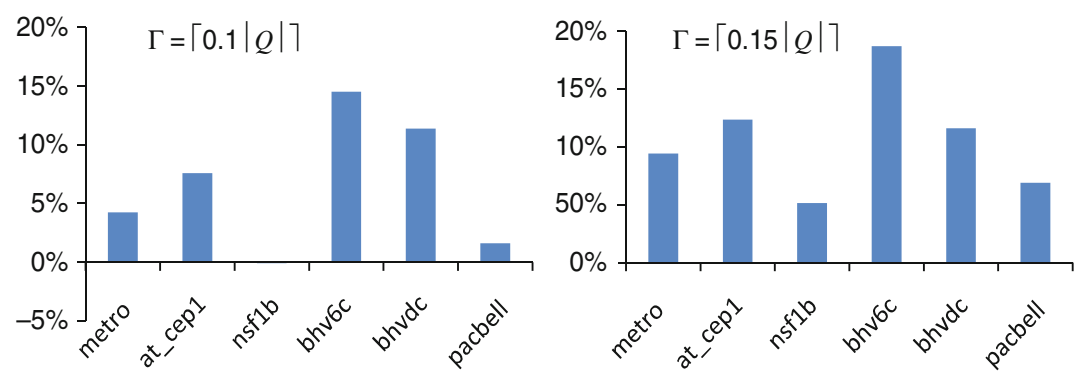

Fig. 6 Savings in design cost by using the hybrid model rather than the BS model

terms of their routing performances. For this purpose, we first generate 20 demand matrices $\dot{d}^{1}, \dot{d}^{2}, \ldots, \dot{d}^{20}$ for each instance where the demand $\dot{d}_{q}^{j}$ for each commodity $q \in Q$ is normally distributed with mean $\tilde{d}_{q}$ and standard deviation $0.5 \tilde{d}_{q}$. Then given the optimal capacity configurations $y(B S)$ and $y(h y b)$, we determine the maximum total flow $F^{j}(B S)$ and $F^{j}(h y b)$ we can route for the demand matrix $\dot{d}^{j}$ for all $j=1, \ldots, 20$ by solving a linear programming problem. For each demand matrix, we calculate the fraction of demand routed for both demand models as $F^{j}(B S) / \sum_{q \in Q} \dot{d}_{q}^{j}$ and $F^{j}(h y b) / \sum_{q \in Q} \dot{d}_{q}^{j}$, respectively. Finally, we take the average over the 20 demand matrices to evaluate the two robust designs. We present our test results in Table 2 where $R_{h y b}$ and $R_{B S}$ are the average routing rates for the hybrid and $B S$ models, respectively, whereas $\Delta_{\text {cost }}$ shows the increase in design cost if the $B S$ model rather than the hybrid model is used. We see that the average routing rates are quite close for metro and at-cepl, whereas they are equal for pacbell. On the other hand, $y(B S)$ is clearly more costly than $y(h y b)$ in all instances. Hence, we can suggest the hybrid model to provide almost the same level of availability at a much lower cost. 
Table 2 Routing rate and cost comparison between the hybrid model and the BS model

\begin{tabular}{lllc}
\hline Instance & $R_{\text {hyb }}(\%)$ & $R_{B S}(\%)$ & $\Delta_{\text {cost }}(\%)$ \\
\hline metro & 95.7 & 97.3 & 9.4 \\
at-cep1 & 96.6 & 97.7 & 14.1 \\
pacbell & 99.9 & 99.9 & 7.4 \\
\hline
\end{tabular}

\section{Conclusion}

In this chapter, we introduced the hybrid model as a new demand uncertainty definition. It inherits the strengths of the two well-known and frequently used demand models: it is easy to specify like the hose model and it avoids over-conservatism like the BS model.

We provided two compact MIP formulations, i.e., $N L P_{h y b}$ and $N L P_{\text {alt }}$, for robust NLP under the hybrid model and compared them in terms of their computational performances. Finally, we discussed how the optimal design cost changes for different demand models. When compared with the interval model, the hose model, and the BS model, we observed that the hybrid model provides significant cost savings by exploiting additional information to exclude overly pessimistic worst-case scenarios. Our test results are encouraging for undertaking further studies on robust network design problems.

\section{References}

1. Altın A, Amaldi A, Belotti P, Pınar MÇ (2007) Provisioning virtual private networks under traffic uncertainty. Networks 49(1):100-115

2. Altın A, Belotti P, Pınar MÇ (2010) OSPF routing with optimal oblivious performance ratio under polyhedral demand uncertainty. Optimiz Eng, 11(3), pp 395-422

3. Altın A, Yaman H, Pınar MÇ (to appear) The robust network loading problem under hose demand uncertainty: formulation, polyhedral analysis, and computations. INFORMS J Comput

4. Atamtürk A (2006) Strong reformulations of robust mixed 0-1 programming. Math Program 108:235-250

5. Atamtürk A, Rajan D (2002) On splittable and unsplittable capacitated network design arc-set polyhedra. Math Program 92:315-333

6. Atamtürk A, Zhang M (2007) Two-stage robust network flow and design under demand uncertainty, Oper Res 55:662-673

7. Avella P, Mattia S, Sassano A (2007) Metric inequalities and the network loading problem. Discrete Optimiz 4:103-114

8. Ben-Ameur W, Kerivin H (2005) Routing of uncertain demands. Optimiz Eng 3:283-313

9. Ben-Tal A, Goryashko A, Guslitzer E, Nemirovski A (2004) Adjustable robust solutions of uncertain linear programs. Mathem Program Ser A 99:351-376

10. Ben-Tal A, Nemirovski A (1998) Robust convex optimization. Math Oper Res 23(4):769-805

11. Ben-Tal A, Nemirovski A (1999) Robust solutions of uncertain linear programs. Oper Res Lett 25:1-13

12. Ben-Tal A, Nemirovski A (2008) Selected topics in robust convex optimization. Math Program112(1):125-158

13. Bertsimas D, Sim M (2003) Robust discrete optimization and network flows. Math Program Ser B 98:49-71

14. Bertsimas D, Sim M (2004) The price of robustness. Oper Res52:35-53 
15. Berger D, Gendron B, Potvin J, Raghavan S, Soriano P (2000) Tabu search for a network loading problem with multiple facilities. J Heurist 6:253-267

16. Bienstock D, Chopra S, Günlük O, Tsai C-Y (1998) Minimum cost capacity installation for multi-commodity network flows. Math Program 81:177-199

17. Bienstock D, Günlük O (1996) Capacitated network design - polyhedral structure and computation. INFORMS J Comput 8:243-259

18. Duffield N, Goyal P, Greenberg A, Mishra P, Ramakrishnan K, van der Merive JE (1999) A flexible model for resource management in virtual private networks. In: Proceedings of ACM SIGCOMM, pp 95-108, Massachusetts, USA.

19. Erlebach T, Rúegg M (2004) Optimal bandwidth reservation in hose-model vpns with multipath routing. Proc IEEE Infocom, 4:2275-2282

20. Fingerhut JA, Suri S, Turner JS (1997) Designing least-cost nonblocking broadband networks. J Algorithm, 24(2):287-309

21. Günlük O (1999) A Branch-and-Cut algorithm for capacitated network design problems. Math Program 86:17-39

22. Günlük O, Brochmuller B, Wolsey L (2004) Designing private line networks - polyhedral analysis and computation. Trans Oper Res 16:7-24. Math Program Ser. A (2002) 92:335-358

23. Gupta A, Kleinberg J, Kumar A, Rastogi R, Yener B (2001) Provisioning a virtual private network: a network design problem for multicommodity flow. In: Proceedings of ACM symposium on theory of computing (STOC), Crete, Greece, pp 389-398

24. Gupta A, Kumar A, Roughgarden T (2003) Simpler and better approximation algorithms for network design. In: Proceedings of the ACM symposium on theory of computing (STOC), pp 365-372, San Diego, CA.

25. Italiano G, Rastogi R, Yener B (2002) Restoration algorithms for virtual private networks in the hose model. In: IEEE INFOCOM, pp 131-139.

26. A. Jüttner, Szabó I, Szentesi Á (2003) On bandwidth efficiency of the hose resource management in virtual private networks. In: IEEE INFOCOM, 1:386-395.

27. Karaşan O, Pınar MÇ, Yaman H (2006) Robust DWDM routing and provisioning under polyhedral demand uncertainty. Technical report, Bilkent University

28. Kumar A, Rastogi R, Silberschatz A, Yener B (2001) Algorithms for provisioning virtual private networks in the hose model. In: SIGCOMM'01, August 27-31, 2001, San Diego, CA, USA

29. Magnanti TL, Mirchandani P (1993) Shortest paths single origin-destination network design, and associated polyhedra. Networks 23:103-121

30. Magnanti TL, Mirchandani P, Vachani R (1993) The convex hull of two core capacitated network design problems. Math Program 60:233-250

31. Magnanti TL, Mirchandani P, Vachani R (1995) Modeling and solving the two-facility capacitated network loading problem. Oper Res 43(1):142-157

32. Mirchandani P (2000) Projections of the capacitated network loading problem. Eur J Oper Res 122:534-560

33. Mudchanatongsuk S, Ordoñez F, Liu J (2008) Robust solutions for network design under transportation cost and demand uncertainty. J Oper Res Soc 59(5):652-662

34. Ordoñez F, Zhao J (2007) Robust capacity expansion of network flows. Networks 50(2): $136-145$

35. Rardin RL, Wolsey LA (1993) Valid inequalities and projecting the multicommodity extended formulation for uncapacitated fixed charge network flow problems. Eur J Oper Res 71:95-109

36. Sim M (2009) Distributionally robust optimization: a marriage of robust optimization and stochastic optimization. In: Third nordic optimization symposium, Stockholm, Sweden.

37. http://sndlib.zib.de/home.action.

38. Soyster AL (1973) Convex programming with set-inclusive constraints and applications to inexact linear programming. Oper Res 21:1154-1157

39. Swamy C, Kumar A (2002) Primal-dual algorithms for connected facility location problems. In: Proceedings of the international workshop on approximation algorithms for combinatorial optimization (APPROX), Lecture Notes in Computer Science series, 2462:256-270

40. Yaman H, Karaşan OE, Pınar MÇ (2007) Restricted robust uniform matroid maximization under interval uncertainty. Math Program 110(2):431-441 